\title{
A cost-benefit approach for the evaluation of prognostics-updated maintenance strategies in complex dynamic systems
}

\author{
Jose Ignacio Aizpurua \& Victoria M. Catterson \\ Department of Electronic \& Electrical Engineering - Institute for Energy \& Environment \\ University of Strathclyde, Glasgow, UK
}

Ferdinando Chiacchio \& Diego D'Urso

Department of Industrial Engineering

Universitá di Catania, Catania, Italy

\begin{abstract}
The implementation of maintenance strategies which integrate online condition data has the potential to increase availability and reduce maintenance costs. Prognostics techniques enable the implementation of these strategies through up-to-date remaining useful life estimations. However, a cost-benefit assessment is necessary to verify the scale of potential benefits of condition-based maintenance strategies and prognostics for a given application. The majority of prognostics applications focus on the evaluation of a specific failure mode of an asset. However, industrial systems are comprised of different assets with multiple failure modes, which in turn, work in cooperation to perform a system level function. Besides, these systems include time-dependent events and conditional triggering events which cause further effects on the system. In this context not only are the system-level prognostics predictions challenging, but also the cost-benefit analysis of condition-based maintenance policies. In this work we combine asset prognostics predictions with temporal logic so as to obtain an up-to-date system level health estimation. We use asset level and system level prognostics estimations to evaluate the cost-effectiveness of alternative maintenance policies. The application of the proposed approach enables the adoption of conscious trade-off decisions between alternative maintenance strategies for complex systems. The benefits of the proposed approach are discussed with a case study from the power industry.
\end{abstract}

\section{INTRODUCTION}

Prognostics is an emerging field with potential economic benefits for maintenance (Vachtsevanos, Lewis, Roemer, Hess, \& Wu 2007). Prognostics approaches predict the Remaining Useful Life (RUL) of the asset under study, which can help to extend the useful life of assets improving availability and reducing maintenance costs (Aizpurua \& Catterson 2015b).

Cost-effective maintenance strategies minimize RUL waste without hazardous consequences (Haddad, Sandborn, \& Pecht 2012). In order to justify the implementation of a maintenance strategy, costbenefit assessments are needed to compare their performance and select the most effective strategy.

In this paper we analyse the cost-effectiveness of different maintenance strategies including reactive, reliability-centred and condition-based maintenance (Kobbacy \& Murthy 2008). In reactive maintenance the asset is repaired after its failure occurrence. Reliability-centred maintenance strategies monitor the failure probability of the asset to de- fine preventive replacement policies based on failure probability thresholds. Condition-based maintenance strategies make use of prognostics predictions to define preventive maintenance actions.

Maintenance strategies can be implemented at different hierarchical levels. While system-level maintenance includes system health information, asset-level strategies concentrate on asset health estimation. In this paper we will focus on asset-level maintenance.

Industrial systems are no longer characterized with static operation logic. Instead, they involve complex interactions that affect the system dependability and include repair/maintenance events for different assets. In this paper we will focus on Dynamic Fault Tree logic to analyse the system failure probability caused by the failure of assets (Manno, Chiacchio, Compagno, D'Urso, \& Trapani 2014).

The main contributions of this paper are the availability-cost assessment of asset-level conditionbased maintenance strategies considering temporal and stochastic dependencies and the comparison among different asset-level maintenance strategies. 
The remainder of this paper is organised as follows. Section 2 presents related work. Section 3 presents the cost-benefit approach for maintenance of dynamic systems. Section 4 applies the proposed approach to a transmission substation case study. Section 5 discusses challenges for system-level maintenance strategies with dynamic scenarios and finally, Section 6 presents conclusions and future prospects.

\section{RELATED WORK}

Different dependencies arise when implementing maintenance strategies for multi-component systems (Thomas 1986). Stochastic dependency implies that the deterioration of an asset impacts the performance of other assets; structural dependency means that maintaining a component implies the maintenance (or unavailability) of other components; and economic dependency addresses the difference between group and independent maintenance actions.

The main goal of maintenance strategies is to maximize availability and minimize risk and cost. To this end, alternative approaches have been presented focused on the optimization of maintenance parameters. Many of the condition-based maintenance approaches propose analytical formulations that integrate degradation models within the maintenance modelling approach and monitor the failure threshold to establish preventive maintenance actions (Grall, Bérenguer, \& Dieulle 2002). Other approaches make use of metaheuristics for the optimization process with additional constraints such as available resources (Camci 2009).

With the advance of prognostics approaches, the interest in condition-based maintenance approaches has increased. (Haddad, Sandborn, \& Pecht 2012) implemented Monte Carlo simulations for the cost-benefit analysis of condition-based maintenance strategies. Options theory is used to evaluate maintenance decisions after RUL prediction, which enable the quantification of the value of waiting to maintain.

Multi-level maintenance strategies have recently gained the interest of researchers as a possibility to reduce maintenance costs by grouping assets. (Nguyen, Do, \& Grall 2015) presents a multi-level preventive maintenance decision making algorithm. At each inspection time, conditional probabilities are calculated to update the system-level reliability modelled with Reliability Block Diagrams (RBD). At component level, a cost-based group improvement factor is used to define preventive maintenance actions. (Do, Vu, Barros, \& Bérenguer 2015) combines corrective and preventive maintenance actions for the optimal scheduling of personnel and optimal grouping of assets. A genetic algorithm is used to integrate limited personnel and downtime durations which can change over time. The system is assumed to be a series model and the failure behaviour of system components is defined by Weibull distributions. (Vu, Do, \& Barros 2016) make use of Birnbaum importance measures and mean residual life for system-level maintenance strategies. Assets are grouped for maintenance depending on if the failure of a component can cause the system failure. RBD logic is used to express the functional/failure operation of the system.

(Do, Voisin, Levrat, \& Iung 2015) evaluates the effect of perfect and imperfect maintenance for condition-based maintenance. An adaptive maintenance policy is proposed to select optimal maintenance actions at each inspection time. Inspection times are based on RUL estimations, so that system failure probability before next inspection remains lower than a threshold. Failure probability between inspection times, imperfect and preventive maintenance thresholds are the optimization variables. Unlimited resources, negligible maintenance durations, and a Gamma degradation process are assumed.

Petri nets have also been used to evaluate the effect of different maintenance parameters on system performance for different maintenance strategies. (Zille, Bérenguer, Grall, \& Despujols 2011) evaluated reliability centred maintenance strategies using Petri nets. Similarly, (Andrews, Prescott, \& Rozires 2014) presented a Petri net model extended with three additional transition types for railway track asset management. Analysed parameters include inspection, renewal, and repair times and maintenance threshold.

All the analysed system-level models take into account the combinatorial failure logic of the system (Nguyen, Do, \& Grall 2015, Do, Vu, Barros, \& Bérenguer 2015, Vu, Do, \& Barros 2016). Besides, most of the reviewed approaches integrate a degradation process to model the degradation of the asset and facilitate the posterior analytical treatment (Grall, Bérenguer, \& Dieulle 2002, Haddad, Sandborn, \& Pecht 2012, Do, Vu, Barros, \& Bérenguer 2015, Do, Voisin, Levrat, \& Iung 2015). This may limit their practicality for different degradation processes.

The main contribution of this paper is the evaluation of prognostics-updated maintenance strategies in complex dynamic systems. These systems are more complex than systems with combinatorial failure logic, because they involve stochastic and temporal dependencies between events. The evaluation of these systems is achieved through the integration of degradation-independent prognostics models with maintenance models. This provides benefits for maintenance modelling enabling the use of different types of degradation processes and prognostics models.

In this paper we will not focus on optimization algorithms because this requires the conception of an analytic formulation for dynamic and repairable systems (see Section 5).

\section{MAINTENANCE MODELS FOR DYNAMIC RELIABILITY SCENARIOS}

Figure 1 shows the proposed multi-component maintenance modelling approach integrating dynamic fail- 
ure logic, prognostics prediction results, and metrics extracted at asset and system level.

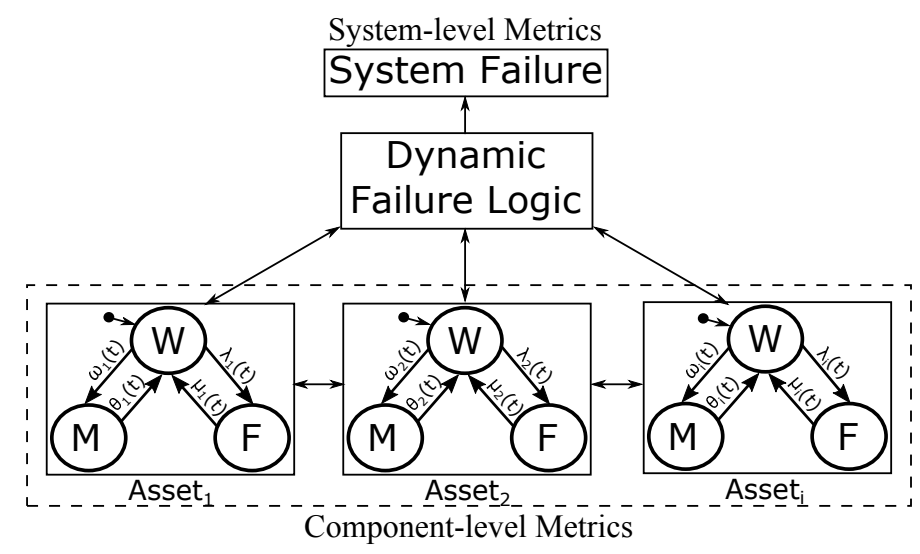

Figure 1: Proposed maintenance approach.

Each asset is modelled with three states. The transition from working $(\mathrm{W})$ to failed $(\mathrm{F})$ state is governed by the failure rate $\lambda(t)$. This failure rate can be updated with up-to-date degradation prediction information. Once the asset fails, it is repaired with a repair rate $\mu(t)$, which models the mean time to repair from failed to working state.

It is possible to avoid the transition to the failed state by performing maintenance prior to the asset failure occurrence. The transition to the maintenance state $(\mathrm{M})$ depends on the maintenance strategy and it is determined by the transition rate $\omega(t)$. Once in the maintenance state, the asset needs a time interval defined by $\theta(t)$ to return back to the working state.

For asset-level reliability centred maintenance strategies $\omega(t)$ is dependent on the asset failure probability. Namely, the asset maintenance is triggered when the failure probability of the asset is above a predefined failure threshold.

As for asset-level condition-based maintenance strategies, $\omega(t)$ is defined as (Aizpurua \& Catterson 2015a):

$\omega(t)=R U L\left(T_{p}\right)-S F$

where $R U L$ is remaining useful life at prediction time $T_{p}$ and $S F$ is a safety factor which integrates uncertainties associated with the RUL prediction.

We calculate the failure probability and cost for each asset as cost-benefit indicators. The failure probability is computed at runtime through the Cumulative Distribution Function (CDF). From basic reliability theory the $\mathrm{CDF}$ is defined as:

$F_{X}(x)=P(X \leq x)=\int_{0}^{x} f_{x}(t) d t$

where $X$ is a random variable of the time to failure and $x$ is the current time instant.

The cost function for each asset $i$ is defined as:

$$
C_{i}(t)=c_{i}+c_{p s d}+c_{d} \times d+c_{u s d}+m_{i}(t)+s_{m}+u_{C B M}
$$

where,

- $c_{i}$ : cost of the asset $i$.

- $c_{p s d}$ : planned shutdown cost due to maintenance.

- $c_{d}$ : downtime cost rate, $d$ : downtime.

- $c_{u s d}$ : unplanned shutdown cost due to failure.

- $m_{i}(t)$ : deterioration cost, $m_{i}(t)=c_{\text {det }} \times \operatorname{Pr}($ fail $)$ and $c_{d e t}$ is the deterioration cost rate.

- $s_{m}$ : initial maintenance setup cost.

- $u_{C B M}$ : cost for updating maintenance plans at runtime (e.g. with prognostics predictions).

Finally, the system-level cost is calculated as the sum of the cost of each asset:

$\operatorname{Cost}_{\text {system }}(t)=\sum_{i=1}^{N} C_{i}(t)$

where $N$ is the total number of assets in the system.

The main assumptions adopted in this paper are: assets are in as bad as old state after repair and availability of unlimited maintenance resources.

We use Stochastic Activity Networks (SAN) (Sanders \& Meyer 2001) to integrate prognostics results, maintenance strategies, dynamic reliability and reconfiguration logic in a single model. SAN is a very flexible formalism which can model complex maintenance strategies. Due to space limitations, in this paper we will emphasize maintenance modelling concepts.

\subsection{Preliminaries on Stochastic Activity Networks}

Stochastic Activity Networks (SAN) extends stochastic Petri Nets generalizing stochastic relationships and adding mechanisms for hierarchical modelling. Figure 2 shows SAN elements (Sanders \& Meyer 2001).

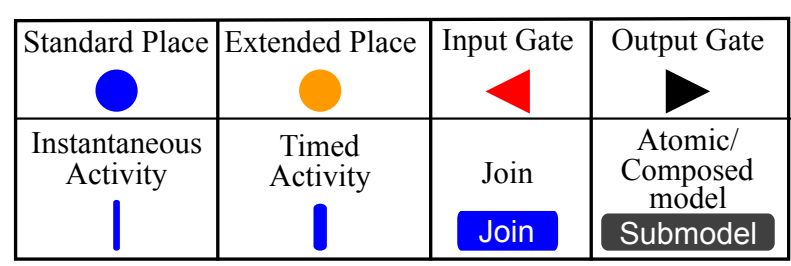

Figure 2: Notation of SAN modelling elements.

Places represent the state of the modelled system. Each place contains a certain number of tokens defining the marking of the place. A standard place contains an integer number of tokens, whereas extended places contain data types other than integers. 
There are two types of activities: instantaneous which complete in negligible amount of time and timed whose duration has an effect on the system performance and their completion time can be a constant or a random value. When it is a random value, it is ruled by a probability distribution function defining the time to fire the activity.

Activities fire based on the conditions defined over the marking of the net and their effect is to modify the marking of the places. The completion of an activity of any kind is enabled by a particular marking of a set of places. The presence of at least one token in each input place enables the firing of the activity removing the token from its input place(s) and placing them in the output place(s). Each activity has a reactivation function that defines when the activity is aborted and a new activity time is immediately obtained from the activity time distribution. The reactivation function provides a mechanism for restarting activities if the reactivation predicate holds for the new marking and for the marking in which the activity was originally activated; and the activity remains enabled.

Another way to enable a certain activity consists of Input Gates (IGs) and Output Gates (OGs). These gates make the SAN formalism general and powerful enough to model complex real situations. They determine the marking of the net based on user-defined $\mathrm{C}++$ rules. IGs control the enabling of activities and define the marking changes that will occur when an activity completes. A set of places is connected to the $I G$ and the $I G$ is connected to an activity. A Boolean condition enables the activity connected to the gate and a function determines the effect of the activity completion on the marking of the places connected to the gate. OGs specify the effect of activity completion on the marking of the places connected to the OG. An output function defines the marking changes that occur when the activity completes.

The SAN models which include the specified SAN elements are modelled in a SAN atomic model. The join operator links through a compositional tree structure different SAN models in a unique composed model. It is possible to link atomic models, composed models, or combinations thereof. In the tree structure, the composed and atomic SAN models are linked through join operators using shared places between the composed and atomic SAN models.

The performance measurements are carried out through reward functions defined over the designed model. Reward functions are defined based on the marking of the net or completion of activities and they are evaluated as the expected value of the reward function. For a complete and formal definition of SAN please refer to (Sanders \& Meyer 2001).

For the sake of clarity, in subsequent figures, we will designate timed activities and we will hide the label of instantaneous activities (cf. Figs. 3-5).

\subsection{Reactive maintenance model}

Reactive maintenance repairs the asset as soon as it fails without preventive maintenance actions. Figure 3 shows the reactive maintenance model comprised of Asset and Control blocks.

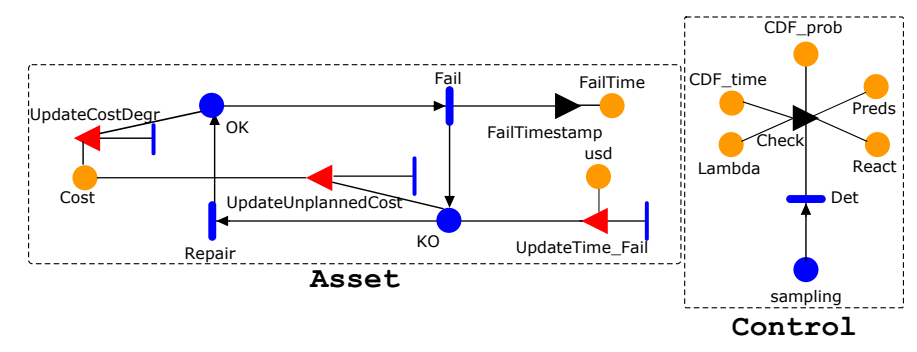

Figure 3: Reactive maintenance model.

The Asset model transits between $\mathrm{OK}$ and $\mathrm{KO}$ places according to Fail and Repair timed activities. The Fail activity is updated (reactivated in SAN terminology) according to prognostics prediction results $(\lambda(t)$ in Fig 1). The FailTimestamp OG calculates the failure time instant and UpdateTime_Fail IG calculates downtime of the asset recorded respectively in the extended places named FailTime and usd. According to Eq. (3), UpdateUnplannedCost and UpdateCostDegr IGs calculate $c_{u s d}$ and $m_{i}(t)$ respectively, both recorded in the Cost extended place.

The core of the Control block is the Check OG. It implements the online CDF calculation and it updates the failure degradation trend with prognostics prediction results. The timed activity Det executes the Check OG at $\Delta \mathrm{t}$ timesteps during the mission time.

On the one hand, for the online CDF calculation, if we assume that the asset under study degrades according to the exponential distribution, Eq. (5) shows the CDF of the exponential distribution:

$F(t)=1-e^{(-\lambda t)}$

where $\lambda$ is the failure rate parameter.

Accordingly, CDF_time and CDF_prob places store the time to failure of the asset and the online failure probability respectively. Note that the constant failure rate can be approximated as the inverse of the RUL $(\lambda \approx 1 / R U L)$ (Banjevic \& Jardine 2006).

On the other hand, the Check OG evaluates if there is any prognostics prediction that needs to be updated according to prognostics prediction results. Prognostics prediction values and prediction time instants are stored in the extended place Preds (cf. Table 1). If the simulation time instant coincides with the prediction time instant $T_{p}$, then the marking of the Lambda extended place is updated with prognostics prediction values and the Fail activity is reactivated with the updated marking of the Lambda extended place.

\subsection{Reliability-centred maintenance model}

Reliability Centred Maintenance (RCM) monitors continuously the failure probability of the asset using 
the PDF integration algorithm (Chiacchio, D'Urso, Manno, \& Compagno 2016), and when it passes a threshold, maintenance is triggered. Figure 4 shows the RCM maintenance model divided into Asset, Control and Maintenance blocks.

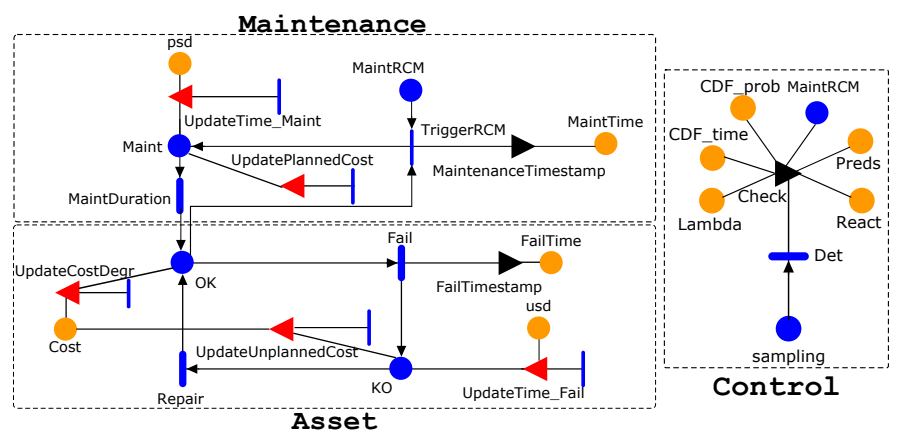

Figure 4: Reliability centred maintenance model.

The Asset block is the same as in the reactive maintenance model. The Control block integrates the MaintRCM place, which is activated when the failure probability modelled by the CDF_prob extended place is above a predefined maintenance threshold.

The Maintenance block performs preventive maintenance according to the MaintRCM place. That is, if the asset is in the OK place and MaintRCM is activated, the asset goes through planned maintenance $(\omega(t)$ in Fig. 1) passing through the Maint place and MaintDuration activity $(\theta(t)$ in Fig. 1). After performing maintenance, the asset returns back to the OK place. The MaintenanceTimestamp OG registers the maintenance time instant and the UpdateTime_Maint IG calculates the planned downtime stored in MaintTime and psd extended places, respectively. Accordingly, the UpdatePlannedCost IG calculates the planned cost due to maintenance.

\subsection{Condition-based maintenance model}

Figure 5 shows the Condition-Based Maintenance (CBM) model divided into Asset, Control, and Maintenance blocks.

The Asset block integrates OK, KO, StandBy and Activate places with Fail and Repair timed activities and an instantaneous activity linking StandBy and Activate places. It updates the system cost via UpdateUnplannedCost and UpdateCostsDegr IGs. Failure instants are calculated with the FailTimestamp $\mathrm{OG}$ and unplanned downtimes are calculated with the UpdateTime_Fail IG. The Fail activity is updated with prognostics prediction results with degradation predictions stored in the Preds place. When the asset is repaired, it remains in the StandBy state until it receives an Activate signal from the reconfiguration mechanism.

The Control block models the system update and online CDF calculation through the Check OG. The Fail and MaintCBM activities $(\lambda(t)$ and $\omega(t)$ in Fig. 1 , respectively) are reactivated and updated with new transition rates via the Lambda and Lambda_M places, respectively. The SF place stores the safety factor to update the maintenance places (cf. Eq. (1)). The CDF_time and CDF_prob places model the online calculation of the CDF according to Eq. (2).

The Maintenance block implements planned shutdown events. The MaintCBM timed activity models the $\omega(t)$ event in Figure 1. MaintCBM has a reactivation logic to update the transition rate to the Maint place according to prognostics prediction results and prediction instants stored in the Preds place (Control block). MaintDuration activity models the $\theta(t)$ event in Figure 1. The UpdateTime_Maint and UpdatePlannedCost IGs calculate planned downtime and cost, respectively.

\section{CASE STUDY}

The correct operation of a transmission substation is critical for power grid performance. Figure 6 shows a configuration example of a transmission substation.

The repair of the transformer is a very expensive and time consuming process (CIGRÉ 2015). Accordingly, the transmission substation is designed to be a fault tolerant system. In the configuration shown in Figure 6, there are always two active transformers and other two are in standby mode. Anytime an active transformer fails, a standby transformer is activated.

Prognostics indicators for transformers and circuit breakers can be extracted from different degradation indicators (e.g. (Catterson, Melone, \& Garcia 2016)) and a suitable prognostics prediction model can be chosen according to a prognostics model selection process (Aizpurua \& Catterson 2015b). However, for simplicity, hypothetical prognostics prediction values displayed in Table 1 are adopted at these prediction instants $\left(T_{p}\right) ; T_{p_{1}}: 6$ years for the circuit breaker; 8 years for the transformer; and $T_{p_{2}}: 12$ years for the circuit breaker; 16 years for the transformer.

Table 1: RUL values (in years) at prediction times $T_{p}$.

\begin{tabular}{ccccccccc}
\hline Assets & $\operatorname{Tr} 1$ & $\operatorname{Tr} 2$ & $\operatorname{Tr} 3$ & $\operatorname{Tr} 4$ & CB1 & CB2 & CB3 & CB4 \\
\hline$T_{p_{0}}$ & 15 & 15 & 15 & 15 & 1 & 1 & 1 & 1 \\
$T_{p_{1}}$ & 12 & 13 & 18 & 20 & 0.6 & 0.5 & 0.9 & 0.9 \\
$T_{p_{2}}$ & 9 & 10 & 16 & 18 & 0.5 & 0.4 & 0.7 & 0.7 \\
\hline
\end{tabular}

For circuit breakers the Asset model in Figures 3 and 4 is implemented and for transformers the Asset model in Figure 5 is implemented with standby states (i.e. as the Component-level Metrics of Fig. 1). The reconfiguration logic implements the priority of transformers. Anytime a transformer fails, the transformer with the highest priority is activated. If a transformer with a higher priority is repaired, it remains in the standby state until a lower priority transformer fails.

For non-repairable systems, reconfiguration strategies can be treated systematically defining at designtime all the possible failure configurations (Aizpurua, 


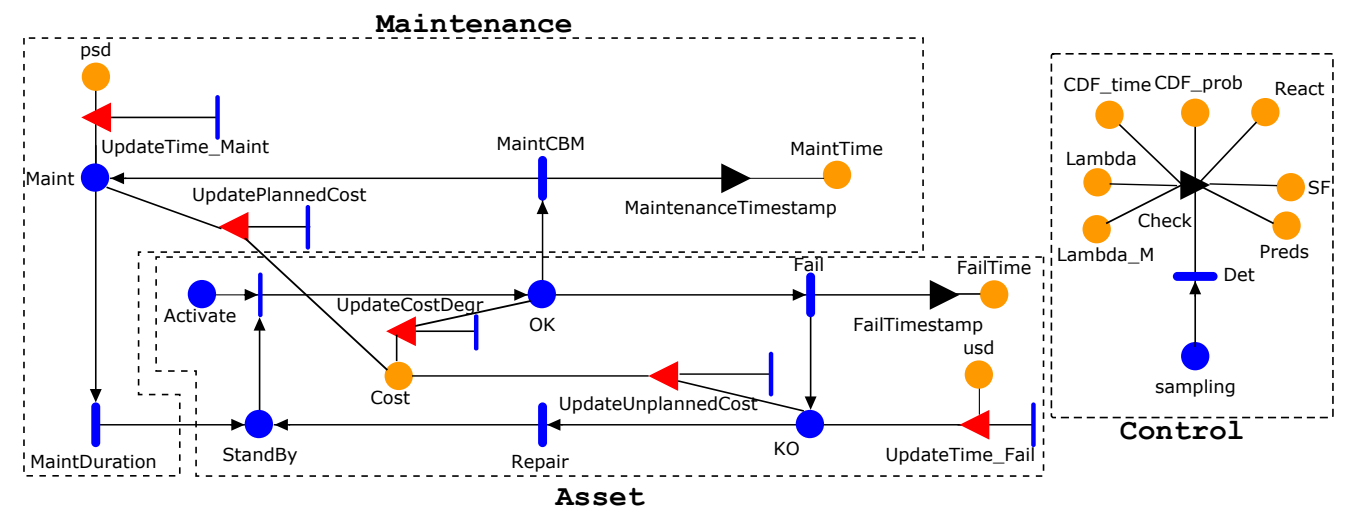

Figure 5: Condition-based maintenance model.

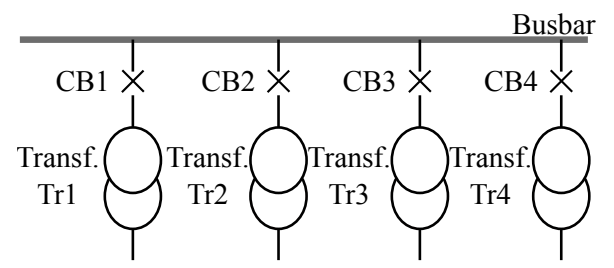

Figure 6: Transmission substation.

Muxika, Papadopoulos, Chiacchio, \& Manno 2016). However, for repairable systems, the number of possible reconfiguration strategies increases due to the stochastic nature of failure and repair events. The reconfiguration logic has been implemented in SAN according to the priority of transformers and possible failure and repair events. Namely, when a standby transformer needs to be activated, the marking of the corresponding Activate place is set (cf. Fig. 5).

The Control and Maintenance blocks depend on the specific maintenance strategy to be analysed.

The failure condition of the transmission substation in Figure 6 can be expressed using Dynamic Fault Tree (DFT) gates (Manno, Chiacchio, Compagno, D’Urso, \& Trapani 2014):

(a) PAND: $Y=P A N D\left(E_{1}, \ldots, E_{N}\right)$; $\mathrm{Y}$ is true iff all events $\left\{E_{1}, \ldots, E_{N}\right\}$ are true and they occur in order: $E_{1} \triangleleft \ldots \triangleleft E_{N}$; otherwise is false (cf. Fig. 7a).

(b) Spare: $Y=S P\left(E_{a c t_{1}}, \ldots, E_{a c t_{M}}, E_{s p_{1}}, \ldots, E_{s p_{N}}\right)$; $\mathrm{Y}$ is true iff all active events $\left\{E_{\text {act }_{1}}, \ldots, E_{\text {act }_{M}}\right\}$ and all spare events $\left\{E_{s p_{1}}, \ldots, E_{s p_{N}}\right\}$ have failed, otherwise is false. Its inputs can be in any of the following three states: standby, working or failed (cf. Fig. 7b).

(c) SEQ: $S E Q\left(E_{1}, \ldots, E_{N}\right) ;\left\{E_{1}, \ldots, E_{N}\right\}$ are true iff all events $\left\{E_{1}, \ldots, E_{N}\right\}$ are true and occur in the following order: $E_{1} \triangleleft \ldots \triangleleft E_{N}$ (cf. Fig. 7c).

(d) FDEP: $\left[E_{1}, \ldots, E_{N}\right]=F D E P(T) ;\left\{E_{1}, \ldots, E_{N}\right\}$ is true if the trigger event $T$ occurs or they fail by themselves; otherwise is false (cf. Fig. 7d).

Figure 8 defines the failure condition of the transmission substation shown in Figure 6 (i.e. Dynamic Failure Logic in Fig. 1), which can be interpreted as follows. The system failure will occur either because:

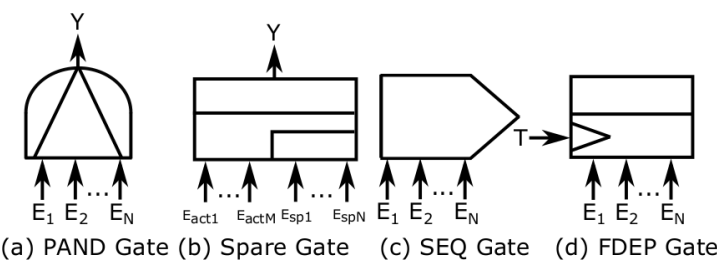

Figure 7: Dynamic Fault Tree gates.

(i) Two transformers fail and two complementary circuit breakers have already failed (IE1-IE6).

(ii) One transformer fails and three complementary circuit breakers have already failed (IE7-IE10).

(iii) All transformers fail (spare gate).

The spare gate determines the activation priority of the inputs from left to right order. That is, any time $\mathrm{Tr}_{1}$ is available, its activation is preferred over the rest of transformers which are in standby state.

We have used the DFT model in Figure 8 to evaluate the system failure probability with prognostics predictions in Table 1 and $\omega=0.8$ yrs; $\theta=1$ yrs (Fig. 1 ).

Table 2 displays analysed maintenance scenarios (SC) with different maintenance strategies and different safety factor (SF) and maintenance threshold values. So as to evaluate the effect of uncertainty on $\mathrm{CBM}$ strategies, we have used different $\mathrm{SF}$ values for transformers $\left(\mathrm{SF}_{\mathrm{Tr}}\right)$ and circuit breakers $\left(\mathrm{SF}_{\mathrm{CB}}\right)$, respecting the proportionality in Table $1\left(\mathrm{SF}_{\mathrm{Tr}}>\mathrm{SF}_{\mathrm{CB}}\right)$. For RCM scenarios we have used two different failure probability values to analyse the effect of replacing assets with different degradation levels.

Table 2: Analysed maintenance scenarios.

\begin{tabular}{ccc}
\hline Scenario & Strategy & Parameters \\
\hline SC1 & Reactive & N/A \\
SC2 & CBM & $\mathrm{SF}_{\mathrm{CB}}=0.3$ year; $\mathrm{SF}_{\mathrm{Tr}}=5$ years \\
$\mathrm{SC} 3$ & $\mathrm{CBM}$ & $\mathrm{SF}_{\mathrm{CB}}=0.1$ year; $\mathrm{SF}_{\mathrm{Tr}}=1$ year \\
$\mathrm{SC} 4$ & $\mathrm{RCM}$ & Maintenance Threshold=0.9 \\
SC5 & $\mathrm{RCM}$ & Maintenance Threshold=0.95 \\
\hline
\end{tabular}

Figure 9 shows the system health state updated with prognostics prediction results in Table 1 for the alternative maintenance scenarios in Table 2. 


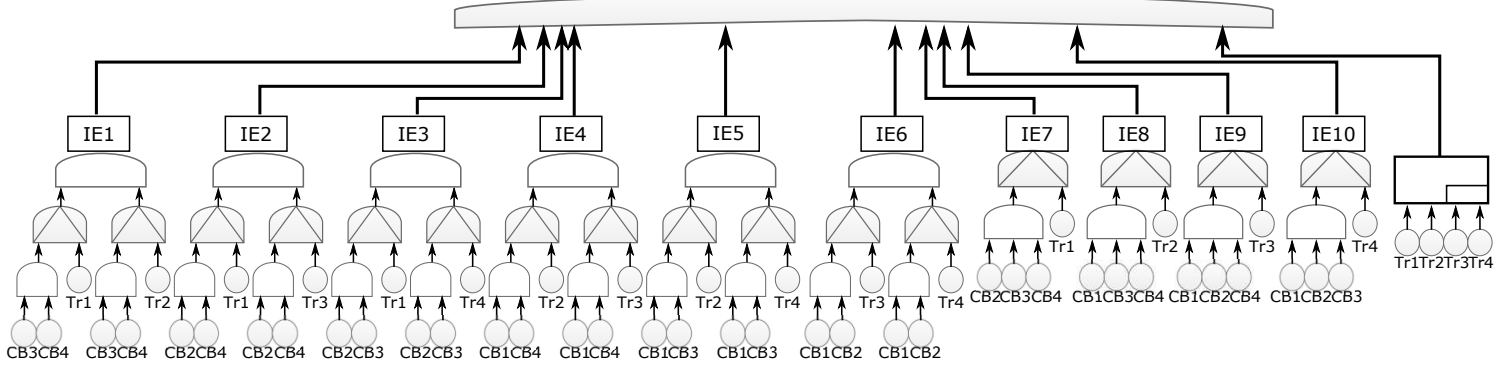

Figure 8: Dynamic Fault Tree model of the transmission substation in Figure 6.

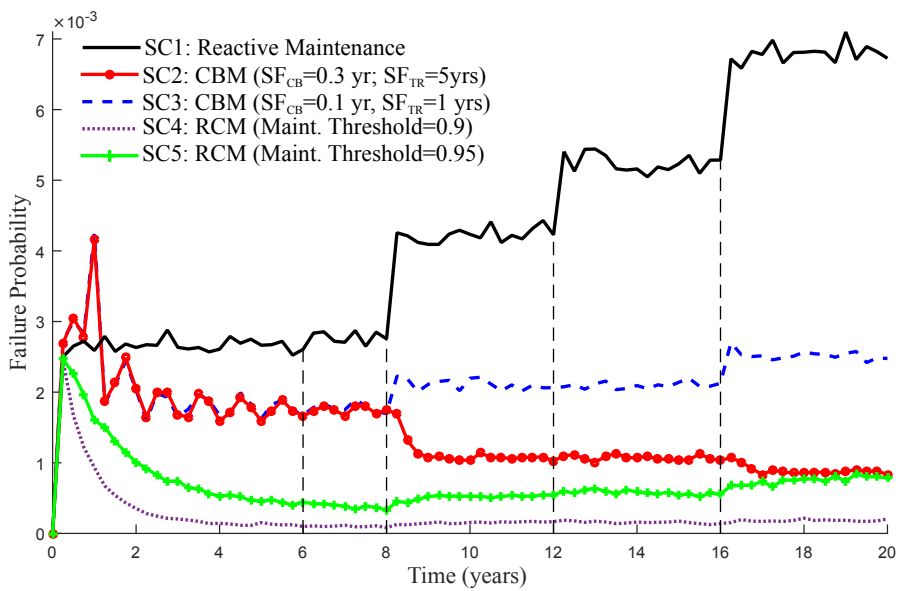

Figure 9: Failure probabilities of maintenance strategies.

With CBM the failure probability of the system is reduced with respect to reactive maintenance because the maintenance is adapted according to new prognostics predictions (cf. Eq. (1)). The effect of the safety factor on the failure probability shows that the bigger the safety margin, the lower the failure probability of the system, i.e. maintenance is done more frequently.

With RCM the system failure probability is even lower than CBM because the maintenance of the asset is performed more frequently. Accordingly, the greater the maintenance threshold, the greater the failure probability because the asset is left to run longer.

For each scenario in Table 2, we have analysed the total cost of the system displayed in Table 3. For each asset we have evaluated its cost according to Eq. (3) and then we have computed the total system cost according to Eq. (4). We have analysed different cost values for cost variables in Eq. (3) and we have assumed as constant the remainder of cost variables not shown in Table 3 (i.e. $c_{i}, s_{m}$, and $u_{C B M}$ ).

As Table 3 displays, the most expensive strategy is the reactive maintenance strategy. On the other hand, the most economic configuration is the CBM strategy. CBM is cheaper than RCM because the maintenance activity is triggered less frequently and the failure probability difference with respect to the RCM strategy is not relevant (cf. Figure 9).

The effect of the deterioration cost rate $\left(c_{\text {det }}\right)$ is also worth mentioning. If we compare cost I and cost IV configurations, variations in $c_{\text {det }}$ and downtime cost rates $\left(c_{d}\right)$ have a representative impact on the final cost. In contrast, if we compare configurations II and
III, we can see that decreasing the downtime cost rate $\left(c_{d}\right)$ impacts slightly on the final cost.

If we compare the cost configurations III and IV, we notice that, if we increase the deterioration cost rate $\left(c_{d e t}\right)$, even decreasing the downtime cost rate $\left(c_{d}\right)$ and unplanned shutdown cost value $\left(c_{u s d}\right)$, the cost of the system increases. The contribution of the deterioration cost rate is more relevant than the downtime cost rate $\left(c_{d}\right)$ and unplanned shutdown cost value $\left(c_{u s d}\right)$.

\section{DISCUSSION}

In this paper, the difference between RCM and CBM strategies resides in the maintenance triggering condition. Both strategies require monitoring the degradation trend of the asset under study. Traditionally, this degradation trend has been considered as a predefined degradation process (see Section 2). However, as shown in this paper, with the recent advance of prognostics approaches, there is room to tailor these maintenance strategies with up to date prognostics prediction results including results coming from different prognostics approaches (see Figure 9).

Transition from asset-level to system-level maintenance strategies is a challenging task with dynamic failure scenarios. An important indicator for decision making of system-level maintenance strategies is to analyse if the failure of an asset can cause the system failure. With static systems this can be evaluated using minimal cut-set concepts (Vu, Do, \& Barros 2016). However, in the context of dynamic repairable scenarios, it is necessary to analyse Minimal Cut Sequence Sets (MCSSs) (Walker \& Papadopoulos 2009).

MCSSs have been analysed for non-repairable DFT gates (Merle, Roussel, \& Lesage 2011). However, the analytic solution for repairable systems is complex and remains unsolved. In this context, the capability to check if the failure of an asset causes the system failure would enable the classification of assets according to their criticality and it would facilitate the conception of maintenance grouping strategies (i.e. economic and structural dependencies, see Section 2).

\section{CONCLUSIONS}

The implementation of cost-effective maintenance strategies is a challenging task for complex systems. 
Table 3: Cost sensitivity analysis at $\mathrm{T}=20$ years.

\begin{tabular}{|c|c|c|c|c|c|c|c|c|c|c|c|c|c|c|c|c|}
\hline \multirow{3}{*}{$\begin{array}{l}\text { Maintenance } \\
\text { Scenarios }\end{array}$} & \multicolumn{4}{|c|}{ Cost Configuration I } & \multicolumn{4}{|c|}{ Cost Configuration II } & \multicolumn{4}{|c|}{ Cost Configuration III } & \multicolumn{4}{|c|}{ Cost Configuration IV } \\
\hline & $c_{d}$ & $c_{p s d}$ & $c_{u s d}$ & $c_{\text {det }}$ & $c_{d}$ & $c_{p s d}$ & $c_{u s d}$ & $c_{d e t}$ & $c_{d}$ & $c_{p s d}$ & $c_{u s d}$ & $c_{\text {det }}$ & $c_{d}$ & $c_{p s d}$ & $c_{u s d}$ & $c_{\text {det }}$ \\
\hline & 1 & 400 & 100 & 1 & 10 & 400 & 400 & 1 & 200 & 400 & 400 & 1 & 100 & 400 & 100 & 10 \\
\hline SC1 & \multicolumn{4}{|c|}{$560,469 \pm 189$} & \multicolumn{4}{|c|}{$1,676,825 \pm 591$} & \multicolumn{4}{|c|}{$1,778,300 \pm 389$} & \multicolumn{4}{|c|}{$2,318,102 \pm 1955$} \\
\hline $\mathrm{SC} 2$ & \multicolumn{4}{|c|}{$176,964 \pm 139$} & \multicolumn{4}{|c|}{$481,604 \pm 555$} & \multicolumn{4}{|c|}{$530,380 \pm 291$} & \multicolumn{4}{|c|}{$879,401 \pm 990$} \\
\hline SC3 & \multicolumn{4}{|c|}{$206,271 \pm 146$} & \multicolumn{4}{|c|}{$579,214 \pm 345$} & \multicolumn{4}{|c|}{$635,408 \pm 298$} & \multicolumn{4}{|c|}{$1,005,978 \pm 1145$} \\
\hline $\mathrm{SC} 4$ & \multicolumn{4}{|c|}{$250,217 \pm 356$} & \multicolumn{4}{|c|}{$728,932 \pm 1555$} & \multicolumn{4}{|c|}{$761,767 \pm 771$} & \multicolumn{4}{|c|}{$1,011,569 \pm 1274$} \\
\hline SC5 & \multicolumn{4}{|c|}{$338,312 \pm 399$} & \multicolumn{4}{|c|}{$984,323 \pm 1109$} & \multicolumn{4}{|c|}{$1,044,655 \pm 1056$} & \multicolumn{4}{|c|}{$1,409,408 \pm 1489$} \\
\hline
\end{tabular}

Current systems integrate not only time-dependent operations, but also inter-dependent mechanisms, where the failure of one asset activates another mechanism. With the recent advance of prognostics approaches, it is possible to implement condition-based maintenance strategies tailored to specific operation conditions. Integrating all these elements, the combination of dynamic failure scenarios and conditionbased maintenance strategies poses new challenges.

In this paper we have integrated prognostics prediction results with Dynamic Fault Tree models and we have analysed different maintenance strategies implemented at the asset level. Stochastic Activity Networks have been used for the evaluation of the cost-effectiveness of different maintenance strategies. Analysed maintenance strategies include reactive, reliability-centred and conditionbased maintenance strategies. In the realized experiments, reliability-centred maintenance strategies show a lower failure probability, but a higher cost compared with condition-based maintenance strategies. Reactive maintenance is the most expensive strategy with the highest failure probability.

In future work we will move from component-level to system-level maintenance strategies, so as to evaluate cost-benefit indicators in complex dynamic systems at different hierarchical levels.

\section{REFERENCES}

Aizpurua, J. I. \& V. M. Catterson (2015a, Dec). On the use of probabilistic model-checking for the verification of prognostics applications. In IEEE International Conference on Intelligent Computing and Information Systems, pp. 7-13.

Aizpurua, J. I. \& V. M. Catterson (2015b, Oct). Towards a methodology for design of prognostics systems. In Annual Conf. of the Prognostics and Health Management Society.

Aizpurua, J. I., E. Muxika, Y. Papadopoulos, F. Chiacchio, \& G. Manno (2016). Application of the D3H2 Methodology for the Cost-Effective Design of Dependable Systems. Safety 2(2), 9.

Andrews, J., D. Prescott, \& F. D. Rozires (2014). A stochastic model for railway track asset management. Reliability Engineering \& System Safety 130, $76-84$.

Banjevic, D. \& A. K. S. Jardine (2006). Calculation of reliability function and remaining useful life for a markov failure time process. IMA Journal of Management Mathematics 17(2), $115-130$.

Camci, F. (2009). System maintenance scheduling with prognostics information using genetic algorithm. IEEE Transactions on Reliability 58(3), 539-552.
Catterson, V. M., J. Melone, \& M. S. Garcia (2016, January). Prognostics of transformer paper insulation using statistical particle filtering of on-line data. IEEE Electrical Insulation Magazine 32(1), 28-33.

Chiacchio, F., D. D’Urso, G. Manno, \& L. Compagno (2016). Stochastic hybrid automaton model of a multi-state system with aging: Reliability assessment and design consequences. Reliability Engineering \& System Safety 149, 1-13.

CIGRÉ (2015). Transformer Reliability Survey. Number 642.

Do, P., A. Voisin, E. Levrat, \& B. Iung (2015). A proactive condition-based maintenance strategy with both perfect and imperfect maintenance actions. Reliability Engineering \& System Safety 133, 22 - 32.

Do, P., H. C. Vu, A. Barros, \& C. Bérenguer (2015). Maintenance grouping for multi-component systems with availability constraints and limited maintenance teams. Reliability Engineering \& System Safety 142, 56 - 67.

Grall, A., C. Bérenguer, \& L. Dieulle (2002). A condition-based maintenance policy for stochastically deteriorating systems. Reliability Engineering \& System Safety 76(2), 167 - 180.

Haddad, G., P. A. Sandborn, \& M. G. Pecht (2012). An options approach for decision support of systems with prognostic capabilities. IEEE Transactions on Reliability 61(4), 872-883.

Kobbacy, K. \& D. Murthy (2008). Complex System Maintenance Handbook. Springer Series in Reliability Eng. Springer.

Manno, G., F. Chiacchio, L. Compagno, D. D'Urso, \& N. Trapani (2014). Conception of Repairable Dynamic Fault Trees and resolution by the use of RAATSS, a Matlab ${ }^{\circledR}$ toolbox based on the ATS formalism. Reliability Engineering \& System Safety 121, 250 - 262.

Merle, G., J.-M. Roussel, \& J.-J. Lesage (2011). Algebraic determination of the structure function of dynamic fault trees. Reliability Engineering \& System Safety 96(2), 267 - 277.

Nguyen, K.-A., P. Do, \& A. Grall (2015). Multi-level predictive maintenance for multi-component systems. Reliability Engineering \& System Safety 144, 83 - 94.

Sanders, W. H. \& J. F. Meyer (2001). Stochastic activity networks: Formal definitions and concepts. In Lectures on Formal Methods and Performance Analysis, Volume 2090 of LNCS, pp. 315-343. Springer Berlin Heidelberg.

Thomas, L. (1986). A survey of maintenance and replacement models for maintainability and reliability of multi-item systems. Reliability Engineering 16(4), 297 - 309.

Vachtsevanos, G., F. Lewis, M. Roemer, A. Hess, \& B. Wu (2007). Intelligent Fault Diagnosis and Prognosis for Engineering Systems. John Wiley \& Sons, Inc.

Vu, H. C., P. Do, \& A. Barros (2016). A stationary grouping maintenance strategy using mean residual life and the birnbaum importance measure for complex structures. IEEE Transactions on Reliability 65(1), 217-234.

Walker, M. \& Y. Papadopoulos (2009). Qualitative temporal analysis: Towards a full implementation of the fault tree handbook. Control Eng. Practice 17(10), 1115-1125.

Zille, V., C. Bérenguer, A. Grall, \& A. Despujols (2011). Modelling multicomponent systems to quantify reliability centred maintenance strategies. Proc. of the Inst. of Mechanical Eng., Part O: Journal of Risk and Reliability 225(2), 141-160. 